\title{
Una nueva especie del género Macraspis Macleay (Scarabaeidae «Pleurosticti»: Rutelinae: Rutelini) de Colombia
}

\author{
A new species of the genus Macraspis Macleay \\ (Scarabaeidae «Pleurosticti»: Rutelinae: Rutelini) from Colombia
}

\author{
Jhon César Neita Moreno*
}

Resumen

Se describe una nueva especie del género Macraspis MacLeay de Colombia, capturada en la Serranía de la Macarena (Meta). Se ilustran los caracteres que la diferencian de especies cercanas. Se incluye un listado con las especies registradas para Colombia.

Palabras clave: Distribución, Machos, Morfología, Nueva especie.

\begin{abstract}
A new species of the genus Macraspis MacLeay of Colombia, captured in the Serranía de la Macarena (Meta) is described. Characters that differentiate of closely related species are illustrated. A list of species recorded for Colombia is included.
\end{abstract}

Keyword: Distribution, Male, Morphology, New species.

\section{Introducción}

A nivel mundial se han reportado 200 géneros y 5.000 especies de Rutelinae agrupadas en siete tribus: Alvarengiini Frey 1975, Anatistini Lacordaire 1856, Anomalini Streubel 1839, Anoplognathini Macleay 1819, Geniatini Burmeister 1844 y Rutelini Macleay 1819 (Bouchard et al. 2011), muy diversificadas en el nuevo mundo. Los escarabajos rutelinos se caracterizan por tener el cuerpo ovalado y robusto, algunas veces estilizado, con el dorso muy convexo, sus patas son fuertes y de longitud variable. Normalmente el borde exterior de la mandíbula no está cubierto por el clípeo. El Labro se proyecta ligeramente más allá del ápice del clípeo (excepto en Anomalacra-Anomalini). Antenas con 9 o 10 artejos, clava antenal con 3 segmentos. Escutelo expuesto. Coxas anteriores transversas. Ápice de las mesotibias con 2 espinas; espinas centrales adyacentes (no separadas por el primer segmento mesotarsal).

La tribu Rutelini es casi de distribución restrin- gida al nuevo mundo. Aproximadamente $80 \%$ de los géneros y $72 \%$ de las especies se distribuyen en esta región (Ohaus 1934; Jameson 1998). Los escarabajos de la tribu Rutelini se caracterizan por la siguiente combinación de caracteres: labro en posición horizontal, casi paralelo con respecto al clípeo y normalmente separado de este por una sutura, antena formada por diez artejos excepto: Parachrysina, Eremophygus, Pseudogeniates richterianus (hembra), y el margen externo de los élitros sin borde membranoso (Morón et al. 1997; Jameson 1998). Dentro de la tribu Rutelini encontramos el género Macraspis MacLeay 1819.

El género Macraspis está representado por 42 especies y 11 subespecies que se distribuyen desde México a Argentina (Soula 1998); los adultos se caracterizan por la siguiente combinación de caracteres: borde anterior del clípeo ligeramente redondeado y levantado; borde exterior de las mandíbulas escotado y bidentado; borde basal del pronoto ligeramente trisinuado; escutelo proporcionalmente gran-

* Universidad Nacional de La Plata, Laboratorio de Sistemática y Biología Evolutiva, La Plata, Buenos Aires, Instituto Argentino de Investigaciones de las Zonas Áridas, CCT-Conicet, Mendoza, Argentina. e-mail: cneitaj@unal.edu.co
Fecha recepción: Marzo 2, 2014
Fecha aprobación: Mayo 22, 2014
Editor asociado: Neita JC 
de, más largo que ancho, los mesoepímeros sobresalen ligeramente frente al húmero elitral; proceso mesoesternal muy largo y grueso, con el ápice un poco dirigido hacia arriba; los metafémures tienen un órgano estridulador dorso-apical; el ápice de las metatibias presenta pequeñas sedas espiniformes. Los machos tienen los protarsos un poco engrosados y la placa pigidial prominente hacia el ápice (Morón y Paucar 1997; Soula 1998). En Colombia se han reportado 11 especies y una subespecie (Neita y Gaigl 2008). Poco se conoce acerca de la historia natural del género; no obstante, sus larvas son de hábitos saprófagos, habitualmente se encuentran en madera en proceso de descomposición tanto de árboles muertos en pie como caídos. De las 42 especies que conforman el género, tan sólo las larvas de siete especies han sido descritas (Morón y Paucar 2003).

El presente trabajo tiene como objetivos describir una nueva especie de Macraspis de Colombia y proporcionar los caracteres diagnósticos que faciliten su identificación.
Macraspis fernandezi Neita n. sp.

Material tipo: Holotipo macho. «COLOMBIA: Meta, San Juan de Arama/Parque Nacional Natural La Macarena, La Curia, Bosque de Galería./ 03 20' 47"N; 7353'22" W. 13 marzo de 1986. /F. Fernández Castiblanco, Leg.

Holotipo depositado en la colección Entomológica del Instituto Alexander von Humboldt, IAvH-N Catálogo 88479.

Descripción: Holotipo macho (Figura1). Longitud: 12,44 mm; ancho humeral: $6,11 \mathrm{~mm}$. Coloración primariamente verde brillante, cabeza dorsalmente brillante; pronoto con un patrón de coloración irregular, coloración anterior verde proyectada hasta el área posterior, reducida en el disco elitral, con una expansión suave hacía los bordes laterales posteriores, coloración amarillo brillante hacía los bordes laterales que se proyecta hacía el disco elitral y área posterior; escutelo verde con los bordes laterales marrón oscuro, élitros verdes; patas y área ventral marrón con reflejos metálicos. Cabeza: Clípeo

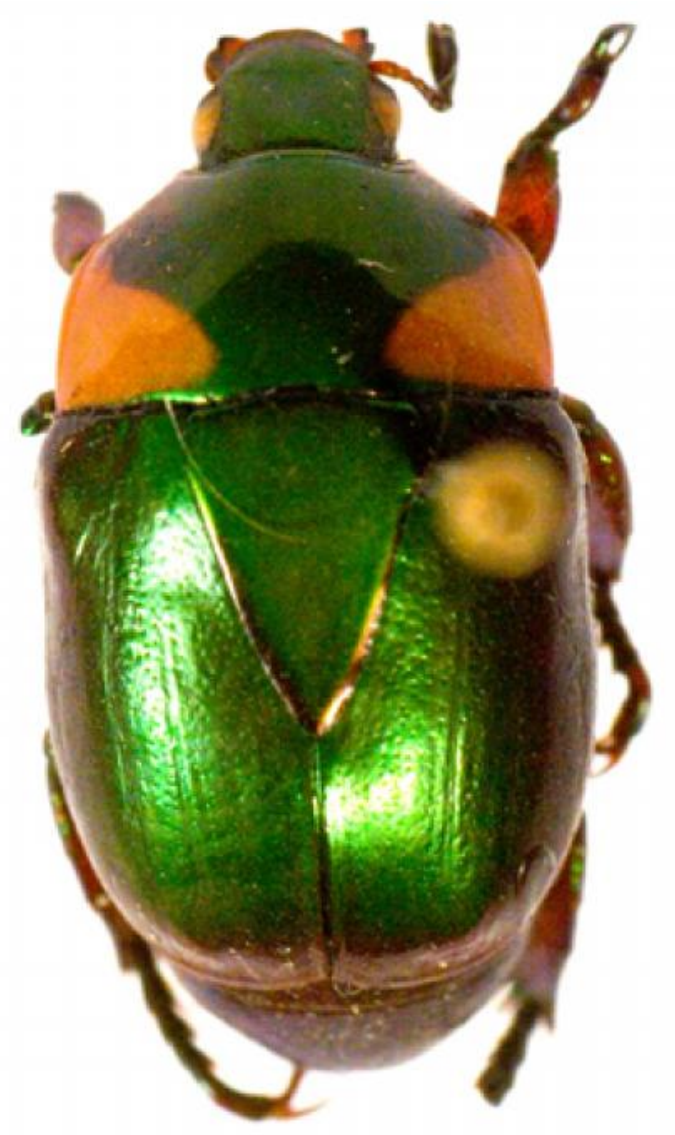

Figura 1. Holotipo macho de Macraspis fernandenzi n. sp. 
redondeado, con puntos fuertemente impresos, ápice ligeramente levantado; sutura fronto-clipeal interrumpida en la mitad, moderamente marcada. Frente con puntos esparcidos, en su parte media ligeramente cóncavo, área basal con puntos escasos, borde lateral de los ojos hacía el cantón ocular con 5 estrías longitudinales. Antena con 10 artejos, clava antenal tan larga como los artejos 2-7 combinados. Mandíbulas con el borde lateral escotado, ápice con el borde externo provisto de un pequeño diente agudo. Pronoto: Ligeramente convexo, anteriormente más estrecho; sin reborde posterior, puntos moderadamente densos e irregularmente distribuidos. Escutelo dos veces más largo que ancho. Élitros: Puntos pequeños, ocelados y densos, liso sin estrías; área humeral hacía la epipleura ligeramente cóncava, ápice elitral redondeado. Pigidio: Más largo que ancho, estrías circulares, sin puntos, sedas ausentes; en vista lateral ligeramente convexo. Patas: Protibias bidentadas, protarsómeros 1-4 más cortos que el $5^{\circ}$, este último engrosado. Uña interna gruesa y plana, con una ligera hendidura en el ápice. Sedas gruesas y cortas en la parte interna de los protarsómeros que se extienden desde la parte basal hasta el ápice. Mesotibias con el ápice truncado, con 8 sedas cortas espiniformes; metatibias con el ápice truncado con 8 sedas cortas espiniformes. Uñas meso y matatarsales externa bífida, la interna simple. Vientre: Mesoesternón con punteaduras fuertes, pequeñas, oceladas, poco densas, proceso mesoesternal desarrollado, con el ápice levantado y redondeado; metaesternón con punteaduras pequeñas escasas, punteaduras alargadas a manera de estrías en su tercio medio, ventritos con estrías laterales, área mesal con punteaduras, cada esternito con una línea de punteaduras transversales setiferas, sedas cortas espiniformes. Pará- meros delgados y largos con una dilatación en ápice con reflejos metálicos a lo largo de los parámeros.

Hembra: Desconocida.

Etimología: Esta especies es dedica al Dr. Fernando Fernández Castiblanco, Mirmecólogo, gran compañero y amigo en la lucha por mejorar el conocimiento de la diversidad entomológica en Colombia; además es la persona que capturó la nueva especie en una de las salidas de campo realizada por el grupo de investigación del Instituto Alexander von Humboldt.

Diagnosis: Esta especie se relaciona con Macraspis lateralis (Olivier 1789). La nueva especie presenta el borde posterior de las mandíbulas redondeados y el borde anterior del clípeo ligeramente parabólico (Figura 2a); por su parte, en M. lateralis las mandíbulas en su borde posterior son casi rectas y el borde anterior del clípeo redondeado (Figura 3a); por otro lado, la nueva especie presenta dos dientes en las tibias anteriores (Figura 2b) y

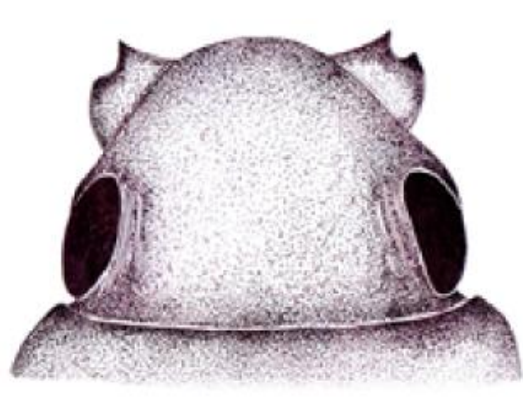

$2 \mathrm{a}$

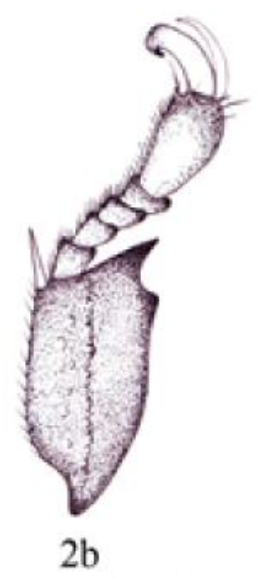

Figuras 2a-b. a. Cabeza (en vista dorsal), b. Protibia y protarsómeros (en vista dorsal) de Macraspis fernandenzi $\mathrm{n}$. sp.

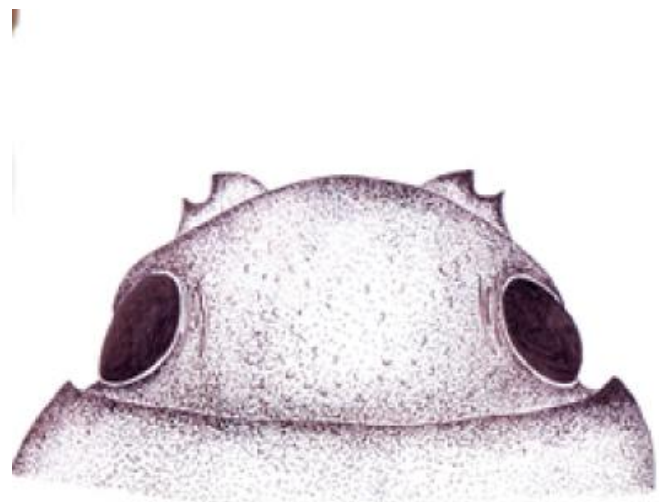

3a

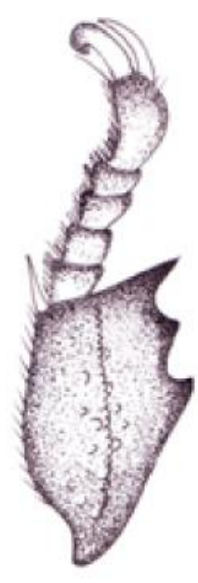

$3 b$
Figuras 3a-b. a. Cabeza (en vista dorsal), b. Protibia y protarsómeros (en vista dorsal) de Macraspis lateralis (Olivier). 


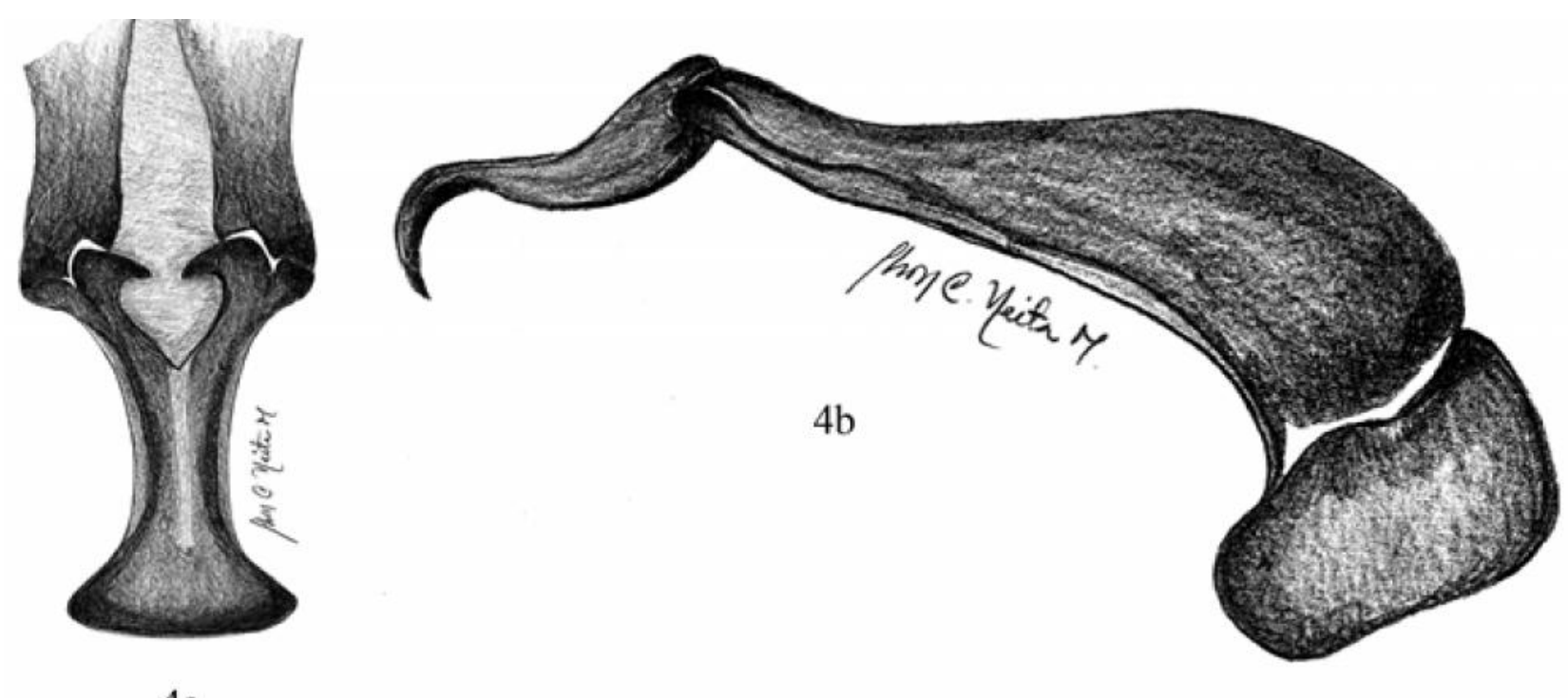

$4 a$

Figuras 4a-b. a. Vista frontal de los parámeros, b. Vista lateral de los parámeros de Macraspis fernandenzi n. sp.

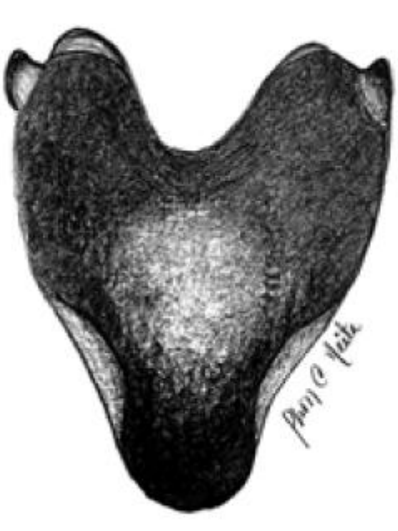

$5 \mathrm{a}$

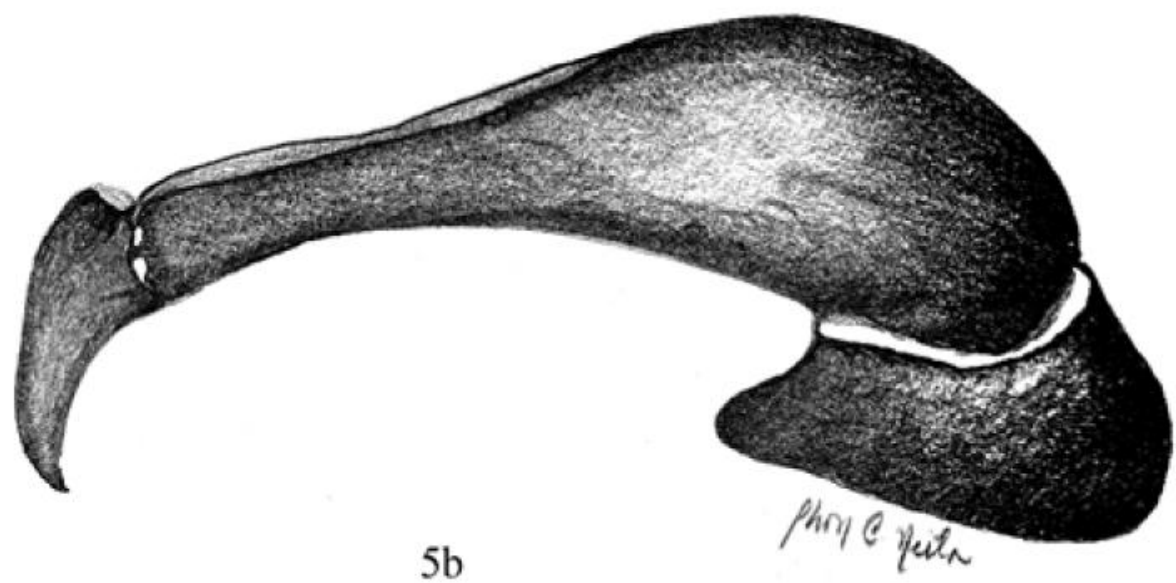

$5 \mathrm{~b}$

Figuras 5a-b. a. Vista frontal de los parámeros, b. Vista lateral de los parámeros de Macraspis lateralis (Olivier).

M. lateralis presenta tres dientes fuertemente desarrollados (Figura 3b). Las genitalias de los machos en ambas especies son completamente diferentes. La nueva especie presenta unos parámeros delgados y largos, constreñidos en su tercio medio, con el ápice ligeramente dilatado (Figura 4a); en vista lateral los parámeros presentan una curvatura hacia el ápice (Figura $4 b$ ). Por su parte, en $M$. lateralis los parámeros tienen forma de corazón, carente de alguna constricción en su tercio medio o dilatación en el ápice (Figura 5a); en vista lateral los parámeros son más robustos y no hay presencia de curvatura (Figura 5b).
Distribución: Esta especie es conocida sólo de la localidad tipo: COLOMBIA: Meta, San Juan de Arama, Parque Nacional Natural La Macarena.

Lista de las especies del género Macraspis MacLeay de Colombia.

Macraspis bouchardi Soula 1998: 31. Departamento: Boyacá.

M. chrysis (Linné 1764): 21. Departamentos: Antioquia, Atlántico, Bolivar, Chocó, Córdoba, Cundinamarca, Risaralda, Sucre, Valle del Cauca.

M. cupripes (Kirsch 1870): 364. Departamento: Caldas. 
M. desmaresti (Waterhouse 1881): 540. Departamento: Antioquia.

M. hirtiventris (Bates 1888): 266. Departamento: Chocó.

M. jamesi Soula 1998: 36. Departamentos: Boyacá, Cundinamarca, Valle del Cauca.

M. melanaria (Blanchard 1850): 206. Departamentos: Caldas, Cundinamarca, Chocó.

M. nazareti Soula 1998: 45. Departamentos: Boyacá, Valle del Cauca.

M. peruviana Ohaus 1898: 52. Departamento: Amazonas.

M. plagiicollis Ohaus 1898: 51. Departamento: Norte de Santander.

M. plicipennis plicipennis Ohaus 1922: 328. Sin datos de distribución.

\section{Agradecimientos}

A Mónica Ospina (ex curadora de la colección Entomológica del Instituto Alexander von Humboldt) y Claudia Alejandra Medina (actual curadora de la colección Entomológica del Instituto Alexander von Humboldt) por el préstamo del material sobre el cual se describe la nueva especie. A Marc Soula (QEPD) por proveerme fotos del tipo de Macraspis lateralis (Olivier).

\section{Literatura citada}

Bouchard P, Bousquet Y, Davies AE, Alonso-Zarazaga MA, Lawrence JF, Lyal CHC. 2011. Family-group names in Coleoptera (Insecta). ZooKeys. 88: 1-972.

Jameson ML. 1998. Phylogenetic analysis of the subtribe Rutelina and revision of the Rutela generic groups (Coleoptera: Scarabaeidae: Rutelinae: Rutelini). Bull Univ Nebraska State Museum. 14: 1-184.

Morón MA, Ratcliffe BC, Deloya C. 1997. Atlas de los escarabajos de México. Coleoptera: Lamellicornia. Vol. 1. Familia Melolonthidae. Subfamilias Rutelinae, Dynastinae, Cetoniinae, Trichiinae, Valginae y Melolonthinae. México, DF: Sociedad Mexicana de Entomología; 280 pp.

Morón MA, Paucar-Cabrera A. 2003. Larvae and pupae of species of the genus Macraspis (Coleoptera: Scarabaeidae: Rutelinae: Rutelini). Can Entomol. 135: 46791.

Neita JC, Gaigl A. 2008. Escarabajos de importancia agrícola en Colombia (Coleoptera: Scarabaeidae «Pleurosticti»). Bogotá: Universidad Nacional de Colombia, Facultad de Agronomía; $160 \mathrm{pp}$.

Ohaus F. 1918. Scarabaeidae Euchirinae, Phaenomerinae, Rutelinae. Coleopterum Catalogus. 20: 1-241.

Ohaus F. 1934. Coleoptera Lamellicornia. Fam. Scarabaeidae, Subfam. Rutelinae. Genera Insectorum, Fasc. 199A: 1219.

Olivier AG. 1789. Entomologie, ou histoire naturelle des insectes, avec leurc caractères génériques et spécifiques, leur description, leur synonymie, et leur figure enlluminée. Coléoptères, Vol. 1. Paris.

Soula M. 1998. Revision des Anthicheirina (sic) 1. Les Coléopterès du Monde. 26: 1-116. 